\title{
SwissDRG und Begleitforschung - sind wir auf Kurs?
}

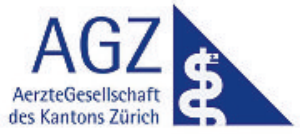

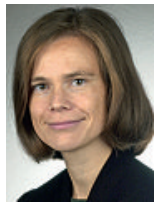

N. Biller-Andorno

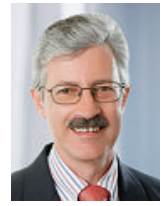

P.-F. Cuénoud

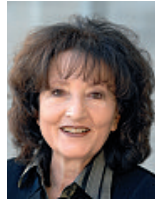

Bea Heim

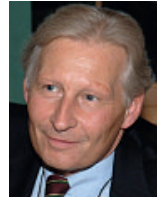

Urs Stoffel

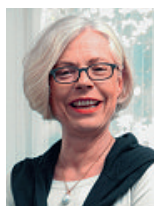

Rita Ziegler

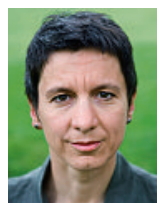

Anna Sax
Die Uhr läuft: Per 1. Januar 2012 soll in der Schweiz das SwissDRG-Fallpauschalensystem zur Abgeltung der stationären Leistungen der Spitäler eingeführt werden. Unter den Fachleuten herrscht Einigkeit darüber, dass eine seriöse Begleitforschung zur Verhinderung bzw. Korrektur von Fehlentwicklungen des neuen Systems unabdingbar ist.

\section{Vielzahl offener Fragen}

Wie wirkt sich die Einführung der DRG auf die ArztPatienten-Beziehung aus? Können die Ärztinnen und Ärzte in den Spitälern noch diejenigen Behandlungen durchführen, die sie für indiziert halten? Nimmt der administrative Aufwand zu? Wie entwickelt sich die Personalsituation? Wie verändert sich die Zusammenarbeit zwischen den verschiedenen Disziplinen sowie zwischen den Ärzten und anderen Gesundheitsfachleuten? Um solche Fragen beantworten zu

\section{Die Podiumsgäste}

Unter der Leitung von SÄZ-Redaktorin Anna Sax, lic. oec. publ., MHA, diskutieren:

- Prof. Dr. med. et phil. Nikola Biller-Andorno, Direktorin des Instituts für Biomedizinische Ethik der Universität Zürich

- Dr. med. Pierre-François Cuénoud, Mitglied des FMH-Zentralvorstands, Verantwortlicher Ressort SwissDRG

Die Veranstaltung wird in Zusammenarbeit mit der Ärztegesellschaft des Kantons Zürich organisiert. Die Durchführung des Anlasses wird möglich dank grosszügiger Unterstützung durch Interpharma, den können, müssten entsprechende Forschungsprojekte vor dem Systemwechsel lanciert werden.

\section{Diskutieren Sie mit}

Wie präsentiert sich die Lage in Sachen Begleitforschung rund ein Jahr vor dem Start ins DRG-Zeitalter? Sind wir auf Kurs, ist das Schiff ins Schlingern geraten oder liegt es gar noch im Hafen und wartet auf günstige Winde? Was ist zu tun, um das Ziel noch rechtzeitig zu erreichen? Zu diesen Fragen diskutieren am nächsten Podiumsanlass der Schweizerischen Ärztezeitung Vertreter der Ärzteschaft mit Fachleuten aus Politik, Ethik, und Verwaltung. Der Einbezug des Publikums in die Diskussion ist zentraler Bestandteil des Konzepts dieser Veranstaltungen, mit denen ein interessanter und konstruktiver Beitrag zur Debatte aktueller Fragen des Gesundheitswesens geleistet werden soll.
- Bea Heim, Nationalrätin Kanton Solothurn

- Dr. med. Urs Stoffel, Co-Präsident der Konferenz Kantonaler Ärztegesellschaften KKA und Präsident der Ärztegesellschaft des Kantons Zürich AGZ

- Rita Ziegler, lic. oec. HSG, Vorsitzende der Spitaldirektion des UniversitätsSpitals Zürich

Verband der forschenden pharmazeutischen Industrie. Die Verantwortung für Konzept und Inhalt des Podiums liegt bei der Schweizerischen Ärztezeitung.

\section{Eintritt frei - Anmeldung erforderlich}

Die öffentliche Podiumsdiskussion mit anschliessendem Apéro findet am Mittwoch, 10. November 2010, im Hörsaal 40 der Universität-Zürich Irchel, Winterthurerstrasse 190, 8057 Zürich, statt. Der Eintritt ist frei, eine Anmeldung ist aber erforderlich. Diese kann bis Freitag, 5. November, via E-Mail an redaktion.saez@emh.ch oder via Fax an 0614678556 erfolgen. Bitte Ihren Namen und die Namen allfälliger Begleitpersonen sowie das Stichwort «Anmeldung zum SÄZ-Podium vom 10. November» angeben. Auch telefonische Anmeldungen sind vormittags unter 0614678572 möglich. 